\title{
The Effects of Macrophages Condition Media on the Vessel-Like Structure Formation of Pre-Vascularized Bone Marrow Mesenchymal Stem Cell Sheets
}

Siqi Long

Sichuan University

Lina Ren

Lanzhou university

Xiaoning Liu

Hangzhou Zhongyi Dental Clinic

Jiamin Shi

Changzhi Medical College

Jiaxin Liu

Lanzhou University

Dongyang $\mathrm{Ma}$

the 940th Hospital of Joint Logistics Support Force of PLA

Ping Zhou

Lanzhou University

Liling Ren ( $\sim$ renlil@lzu.edu.cn )

Lanzhou University

\section{Research Article}

Keywords: Bone marrow mesenchymal stem cells, cell sheet, angiogenesis, macrophages

Posted Date: October 27th, 2021

DOI: https://doi.org/10.21203/rs.3.rs-1014071/v1

License: (c) (i) This work is licensed under a Creative Commons Attribution 4.0 International License.

Read Full License 
The effects of macrophages condition media on the vessel-like structure formation of pre-vascularized bone marrow mesenchymal stem cell sheets

Siqi Long ${ }^{\mathrm{a}}$, Lina Ren ${ }^{\mathrm{b}}$, Xiaoning Liu ${ }^{\mathrm{c}}$, Jiamin Shi ${ }^{\mathrm{d}}$, Jiaxin Liu ${ }^{\mathrm{b}}$, Dongyang Ma ${ }^{\mathrm{e}}$, Ping Zhou ${ }^{\mathrm{b} *}$, Liling Ren $^{\mathrm{b} *}$

${ }^{a}$ West China School of Stomatology, Sichuan University, No.14, Section 3, Renmin South Road, Wuhou District, Chengdu,610065, PR China

${ }^{\mathrm{b}}$ School and Hospital of Stomatology, Lanzhou University, No.222 Tianshui South Road, Chengguan District, Lanzhou 730000, Gansu Province, PR China.

${ }^{\mathrm{c}}$ Hangzhou Zhongyi Dental Clinic, No.170, Zhonghe Middle Road, Shangcheng Distict, Hangzhou, Zhejiang, 310000, PR China

${ }^{d}$ Department of Laboratory Animal Centre, Changzhi Medical College, No.161 Liberation East Street, Luzhou District, Changzhi 046000, Shanxi Province, PR China.

e Department of Oral and Maxillofacial Surgery, the 940th Hospital of Joint Logistics Support Force of PLA, Lanzhou, Gansu, 730052, PR China

*Address correspondence to Prof. L.L. Ren (e-mail: renlil@1zu.edu.cn) or to Prof. P. Z (email: j.chen@pku.edu.cn).

\section{Abstract \\ Background}

With the development of tissue engineering and regenerative medicine, engineered tissue constructs have become more prevalent and are now used as substitutions of patients' damaged organs or tissues. To date, a scaffold-free cell transplantation technique based on cell sheets have been widely used in clinic. While poor vascularization and adverse immune reactions to biomedical devices remain crucial challenges in tissue engineering. The inflammatory response represents one of mainly and important regulators of vascularization and engineered tissue function restoration. This study investigated the effect of macrophage secretion on the formation of microvascular-like structures of human 
umbilical vein endothelial cells (HUVECs) in the bone marrow mesenchymal stem cell (BMSC) sheets.

Methods

Researchers differentiated the human monocytic leukemia cell line (THP-1) cells into the resting state of macrophage (M0) by phorbol 12-myristate 13-acetate (PMA) and further activated them into pro-inflammation and pro-healing states with the use of various cytokines. Condition media derived from kinds of macrophages were used to investigate the impact of macrophage on the viability and arrangement of HUVECs on BMSC sheets. Cytokines related to angiogenesis were detected in the culture supernatant of HUVECs, BMSC sheets, and pre-vascularized sheets.

Results

Results indicated that macrophages may guide the arrangement of endothelial cell through the paracrine pathway. The cell sheets that were cultured in the microenvironment with pro-inflammatory macrophages had fewer cell layers compared to those generated in other media. Furthermore, after experiencing high levels of vascular endothelial growth factor (VEGF) and tumor necrosis factor alpha (TNF- $\alpha$ ) in the first 3 days while high level of platelet-derived growth factor (PDGF)-BB but low TNF- $\alpha$ and VEGF concentration in the next 4 days, pre-vascularized sheets had the most significant micro-vessel network.

\section{Conclusions}

The fates of pre-vascularized sheets regulated by macrophages secreted factors in microenvironment and sequential released cytokines could even promote 
neovascularization and angiogenesis.

Keywords: Bone marrow mesenchymal stem cells, cell sheet, angiogenesis, macrophages

\section{Background}

With synergistic progress in fields of tissue engineering and regenerative medicine, kinds of bioengineered substitutes had been generated. Engineered tissue constructs have become a useful tool to improve the lives of patients[1]. However, there are many obstacles to the application of biomaterials in the clinic including adverse immune reactions and poor vascularization[2, 3]. Any implantable device intended to replace damaged tissue and drive regeneration of cells will need to interact with the native cells of the body as well as the immunological and inflammatory responses which precede angiogenic processed[4]. As research progresses toward developing immunomodulatory biomaterial, the understanding of biomaterial-immune system interaction need to be further investigated.

The implantation of biomaterials is accompanied by the destruction of blood vessels and the occurrence of inflammation response. Many studies have been done in an attempt to understand the precise functions of each immune component when responding to the implantation of a novel biomaterial that affects the regeneration process[5]. Macrophages are the best studied cell type and are commonly considered the key point because of their high plasticity and flexibility[6]. During the chronic response to biomaterial, specific proinflammatory macrophages (M1) increase in the implant site. Based on other stimulations, the macrophages can polarize to a pro-healing phenotype (M2). When this switch is happened, the whole microenvironment is stimulated towards regeneration of the injured 
site[1]. Macrophages often include three main subtypes: naïve (M0), pro-inflammatory (M1), or anti-inflammatory (M2). Sometimes M2 macrophages are further categorized into M2a, M2b, and M2c[7]. It has been demonstrated that both M1 and M2 macrophages have proangiogenic contributions. M1 macrophages secrete the highest levels of VEGF, the most potent stimulator of angiogenic processes[8]. M2a macrophages secrete the highest levels of growth factor of PDGF-BB, that can stabilize pericytes and enhance the recruitment of ECs and assembly into vessels[8]. M2b macrophages have immunomodulatory activities and defined as regulatory macrophages[9]. M2c macrophages secrete the highest levels of Matrix metalloproteinase-9 (MMP-9), an important protease involved in the remolding process of vascular beds[8]. Recent studies revealed that both M1 and M2 macrophages play different but vital roles in tissue repair[10].

Cell sheet technology is a scaffold-free approach to generating 3D tissues, that protects cells within their own extracellular matrix[11]. The latest research showed the 3D cell sheet structure could increases MSC paracrine activity as well as upregulates cell-cell, cell-ECM, and gap junction interactions[12]. In previous studies, researchers successfully fabricated pre-vascularized cell sheets and used these sheets to repair bone defects[13, 14]. To promote this technology from bench to bedside, the localized inflammatory response elicited by the pre-vascularized cell sheets is worthy of further exploration. Sukho et al. found that cell sheets formed by adipose tissue-derived or bone marrow-derived stromal cells both are able to stimulate macrophage into the anti-inflammatory phenotype[15]. He et al. discovered that different types of macrophages and their condition media can affect 
the cellular behaviors of BMSCs[16]. Few studies, however, have been done to explore the effect of macrophages on the pre-vascularized sheets. In this study, researchers induced THP-1 cell lines into M0 by PMA and then activated them into pro-inflammation and prohealing states by using various cytokines. Condition media (CM) derived from different types of macrophages was used to investigate the impact of macrophages on the viability and arrangement of endothelial cells on BMSC sheets. Cytokines related to angiogenesis were detected in the culture supernatant of HUVECs, BMSC sheets, and pre-vascularized sheets. The aim of this study is to clarify the relationship between macrophages secretions and vessel-network formation on cell sheets.

\section{Methods}

\section{Macrophage culture and polarization}

THP-1 cells as a model for human monocytes are classically activated by the differentiation agent PMA. THP-1 (American Type Culture Collection, ATCC) were cultured in macrophage media consisting of RPMI-1640 medium supplemented with the following: 10\% FBS, 1\% penicillin-streptomycin, and 0.05mM 2-mercaptoethanol (Sigma). Media was incubated in an atmosphere of $5 \% \mathrm{CO}_{2}$ at $37^{\circ} \mathrm{C}$. The culture started at $2-4 \times 10^{5}$ viable cells $/ \mathrm{ml}$ and was sub cultured when cell concentration reached $8 \times 10^{5}$ cells $/ \mathrm{ml}$. There is currently no standardized protocol for induction of THP-1 cells into macrophage by PMA. Based on the published differentiation protocols $[17,18]$, the researchers designed different experimental conditions to get the best induction conditions for this research. To explore the optimal induction concentration, researchers set four concentrations of PMA: $5 \mathrm{ng} / \mathrm{ml}$, 
$50 \mathrm{ng} / \mathrm{ml}, 100 \mathrm{ng} / \mathrm{ml}$, and $200 \mathrm{ng} / \mathrm{ml}$. These were used to induce two different concentrations of THP-1 cells, $1.0 \times 10^{5}$ cells $/ \mathrm{ml}$, and $2.5 \times 10^{5}$ cells $/ \mathrm{ml}$ into macrophages for $48 \mathrm{~h}$ or $72 \mathrm{~h}$. According to CCK-8 results and the calculated cell densities, shown in Figure S1, $100 \mathrm{ng} / \mathrm{ml}$ PMA was used to induce $2.5 \times 105$ cells $/ \mathrm{ml}$ of THP-1 cells into macrophages (M0) for $48 \mathrm{~h}$. After differentiation, the adherent cells were washed twice with RPMI-1640 medium and left to rest for another $24 \mathrm{~h}$ in the culture medium without PMA in order to obtain the resting state of the macrophage (M0). The pro-inflammatory macrophages, or M1 macrophages, were produced by exposing the M0 phenotype to a medium that contained IFN- $\gamma(20 \mathrm{ng} / \mathrm{ml})$ and LPS $(100 \mathrm{ng} / \mathrm{ml})$ for $24 \mathrm{~h}$. The M0 phenotype was treated overnight in macrophage serum-free medium containing interleukin-4 (IL-4) $(20 \mathrm{ng} / \mathrm{ml})$ to obtain alternatively activated anti-inflammatory, or M2 macrophages.

\section{M0, M1, M2 derived condition medium preparation}

After deriving the M0, M1, and M2 phenotypes from the initial THP-1 cell, removal of stimuli from cultures of cells by washing with PBS. Culture medium without stimuli was added. Culture was continued for another $24 \mathrm{~h}$, and then the supernatant of culture medium was then collected and centrifuged at $400 \mathrm{~g}$ for $10 \mathrm{~min}$. Samples were filtered through 0.22 $\mu \mathrm{m}$ pore filters (Millipore, USA) and frozen at $-80^{\circ} \mathrm{C}$ until they were used for culture of endothelial cells and cell sheets as condition medium (CM). According to different generations, CMs were termed CM (M0), CM (M1), and CM (M2).

\section{Real-time PCR}

The M0, M1, and M2 cells were placed in $75 \mathrm{~cm}^{2}$ cell culture plates and later harvested by 
2 milliliters ( $\mathrm{ml}$ ) of Trizol reagent (Gibco, USA) per plate for RNA isolation. The samples were thawed on ice, and RNA was isolated from the samples by following the chloroformisopropanol precipitation method. RNA was isolated in $40 \mu \mathrm{L}$ of diethyl pyrocarbonate (DEPC) water and stored at $-80^{\circ} \mathrm{C}$ until samples were used for reverse transcription. The quantity of RNA was measured using the Nanodrop 8000 Spectrophotometer (Thermo Scientific) and considered to be pure if the ratio of absorbance at $260 \mathrm{~nm} / 280 \mathrm{~nm}$ was greater than or equal to two. The Revert Aid TM First Stand cDNA Synthesis Kit was used to convert RNA into cDNA. Quantitative real-time polymerase chain reaction (RT-PCR) was

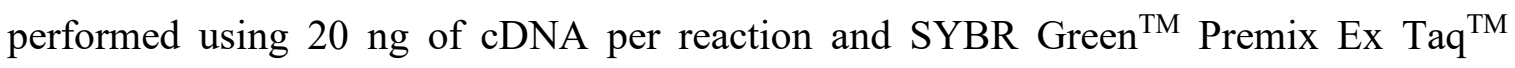
(Takara, Japan) via an ABI 7500 RT-PCR machine (Applied Biosystems by Life Technologies, USA). Three replicate samples were used for each sample. Each replicate was tested in three independent experiments. GAPDH mRNA was used as an internal control. Gene expression values were calculated using the mean $\mathrm{C}_{\mathrm{T}}$ values of the samples. The primer sequences of these genes are shown in Figure 1A.

\section{Scanning electron microscope analysis}

M0, M1, and M2 cells were induced in 24-well plates with slides. Plates were treated with $2.5 \%$ glutaraldehyde and fixed for $24 \mathrm{~h}$. Researchers then washed the plates with the following sequence of reagents: $30 \%$ ethanol for $10 \mathrm{~min}, 50 \%$ ethanol for $10 \mathrm{~min}, 70 \%$ ethanol for $10 \mathrm{~min}, 90 \%$ ethanol for $10 \mathrm{~min}$, and anhydrous ethanol for more than $10 \mathrm{~min}$. Samples were then removed from plates and dried in an ultra-vacuum for more than $4 \mathrm{~h}$ and gold-plating for the 40s. Samples were made available for SEM (Hitachi S-3400N) for 
pictures and further observation.

\section{Fabrication of pre-vascularized cell sheets}

BMSCs were purchased from ScienCell (Catalog Number 7500; Lot Number 6890; USA) and expressed CD73, CD90, and CD105. BMSCs were then cultured in mesenchymal stem cell medium (MSCM). HUVECs were cultured in Endothelial Basal Medium (EBM). Catalog Number 1001; Lot Number 8678; ScienCell; USA Both types of cells were incubated in an atmosphere of $5 \% \mathrm{CO}_{2}$ at $37^{\circ} \mathrm{C}$. BMSCs were placed in six well plates at a density of $9 \times 10^{4}$ cells $/ \mathrm{cm}^{2}$ per well in MSCM. When BMSCs reached $80 \%$ confluence, media was changed to cell sheet culture medium consisting of $90 \%$ high-glucose Dulbecco's modified eagle medium (hDMEM), 10\% fetal bovine serum (FBS), 1\% penicillin-streptomycin, and $100 \mathrm{mg} / \mathrm{ml}$ vitamin $\mathrm{C}$ to promote the production of the extracellular matrix. After 10 to 14 days of culturing, the cell sheets were harvested. In order to engineer pre-vascularized cell sheets, the suspension of HUVECs was then seeded onto the BMSC sheets at a density of $5 \times 10^{4} \mathrm{~cm}^{2}$ in a mixed medium. This medium contained EBM and hDMEM (1:1, v/v). Medium was changed every three days.

\section{Cell counting kit-8 (CCK8) assay}

After fabricating the BMSC sheets and pre-vascularized cell sheets, the medium was changed to a mixed medium containing normal medium and CM $(2: 3, v / v)$. HUVECs $(5 \times$ 104/cm2) were seeded on Matrigel in a 6-well plate with EBM complete medium overnight and then medium was changed to condition media. The CCK-8 assay was performed, and the medium was collected at the time points of 1,3, and 7 days. According to the protocol, 
a 1:10 (v/v) diluted CCK-8 reagent $(500 \mu \mathrm{l} /$ well $)$ was added to the culture plates after moving the medium, followed by incubation at $37^{\circ} \mathrm{C}$ for one to two hours. The supernatant was then transferred to new 96-well plate, $100 \mu$ per hole with four double holes in each group. The optical density (OD) at $450 \mathrm{~nm}$ was determined using a microplate reader.

\section{H\&E staining}

After culturing in macrophage CMs at 1,3, and 7 days, pre-vascularized cell sheets were harvested and immobilized overnight in 4\% paraformaldehyde. Sheets were then rinsed, dehydrated, embedded in paraffin, and cut into $5 \mu \mathrm{m}$ sections. Sectoins were stained with hematoxylin and eosin, then dehydrated with anhydrous ethanol, and then sealed with neutral gum. Images were taken under an optical microscope (Nikon Eclipse E100, Japan).

\section{Immunofluorescence}

The immunofluorescent staining of CD31 was used to characterize the vascularized pattern of HUVECs on BMSC sheets. First, paraffin sections were deparaffinized by treatment with xylene for $30 \mathrm{~min}$ and anhydrous ethanol for $10 \mathrm{~min}$. This was followed by immersing sections in $85 \%$ alcohol for five minutes and $75 \%$ alcohol for five minutes. After deparaffinization, sections were put in EDTA antigen retrieval buffer ( $\mathrm{pH} 9.0)$, placed in a microwave oven, and heated to $95^{\circ} \mathrm{C}$ for $40 \mathrm{~min}$. Sections were then cooled for $20 \mathrm{~min}$ at room temperature and washed with PBS ( $\mathrm{pH} 7.4)$. The following antibodies were used: mouse anti-human CD31 primary antibody (ab9498, Abcam Inc, USA) and the goat antimouse secondary antibody conjugated with AlexaFluor®594 (Invitrogen, USA). Finally, cell nuclei were counterstained with DAPI. After culturing the HUVECs $(5 \times 104 \mathrm{~cm} 2)$ on 
Matrigel in different condition media for 1,3, and 7 days, the arrangement of the nuclei of HUVECs were shown by DAPI staining. Stained samples were then photographed by a fluorescent microscope.

\section{ELISA assay}

The levels of cytokines related to the formation of vascular were measured in culture mediums at the time points of one, three, and seven days. Highly sensitive ELISA kits for Human VEGF and TNF- $\alpha$ (Cloud-Clone Corp, USA) and human PDGF-BB ELISA kit (Multi science, China) were used according to the manufacturer's instructions.

\section{Statistical analysis}

All quantitative data are expressed as the mean \pm standard deviation. Statistical analysis was performed with one-way analysis of variance (ANOVA) followed by Tukey's posttest or two-way ANOVA using GraphPad Prism 8.0 software (San Diego, CA, USA).

Statistical significance was considered at $p<0.05\left({ }^{*} p<0.05,{ }^{* *} p<0.01\right.$, and ${ }^{* * *} p<$ $0.001)$.

\section{Results}

\section{Characterization of polarized macrophages}

Figure 1A shows cell morphologies of macrophages. It can be seen that THP-1 cells adhered to culture plates and differentiated into macrophage phenotypes with marked morphological changes like becoming flat and amoeboid[19]. The M1 phenotype showed a more spindle-like shape when compared to the M2 phenotype[20]. Gene expression was analyzed to identify the specific markers by different macrophage phenotypes. Figure1 B 
shows the PCR primers used in this study. All of these results are reflected in Figure1 C. THP-1 macrophages strongly expressed the monocyte cell maker gene CD14 $(* * p<0.01)$. Specific M1 markers $T N F-\alpha, I L-1 b$, and $C C R 7$ were found in significantly higher levels in cells that were polarized with LPS plus IFN- $\gamma$ as compared to those polarized with IL-4 $(* p<0.05)$. In addition, the expression level of M2 specific markers like CD206 was relatively upregulated in cells that were exposed to IL-4 as compared to those exposed to LPS plus IFN- $\gamma$. The expression of angiogenic factor $V E G F A$ was highest in M1 macrophage group $\left({ }^{*} p<0.05\right)$. There was no significant difference of expressions of CCL18 and PDGF-BB between the two stimulating culture conditions $\left({ }^{*} p<0.05\right)$.

\section{Effect of CMs on the arrangement and viability of endothelial cells}

As shown in Figure S2, the HUVECs in normal EBM were found to have a cobblestonelike morphology, and cells were sparsely aligned. In comparison, the HUVECs in condition media appeared to be slender and tightly arranged on the first and third day, but became smaller on the seventh day. The arrangement of HUVECs nuclei was further observed under an immunofluorescence microscope (Fig. 2A). The nuclei were found to be arranged in small, round circles in control medium, while nuclei were arranged in long circles in all types of CM. As time passed, the nuclei showed a random and disordered arrangement in EBM. On the seventh day of observation, nuclei in CMs aligned in small circles. According to the CCK-8 results (Fig. 2B), the viability of HUVECs in CMs was slightly increased on the first day, but followed by a decrease on the third and seventh days as compared to the control group. Overall, HUVECs were more active in $\mathrm{CM}(\mathrm{M} 1)$ than in $\mathrm{CM}(\mathrm{M} 2) .\left({ }^{* *} p<\right.$ 
0.01 or $\left.^{* * *} p<0.001\right)$. These results suggest that macrophage condition mediums could affect the viability and arrangement of HUVECs.

\section{Effect of CMs on the viability of BMSC sheets, and pre-vascularized cell sheets}

After ten to fourteen days of culturing, the BMSCs formed a dense and continuous cell sheet that could be lifted with forceps. CCK-8 assays were performed to investigate the proliferative capacity of the BMSC sheet and pre-vascularized cell sheets in condition media or normal medium. Figure $3 \mathrm{~A}$ shows that the ascending trend of BMSC cell sheets growth could be observed in all CMs on day 1 and day 3. After 7 days of culturing, compared to the control group, the OD value of BMSC cell sheets in CM(M1) was lower but still higher in both $\mathrm{CM}(\mathrm{M} 0)$ and $\mathrm{CM}(\mathrm{M} 2)$. For pre-vascularized cell sheets (Fig. 3B), the $\mathrm{CM}(\mathrm{M} 0)$ and $\mathrm{CM}(\mathrm{M} 2)$ were able to improve the viability of sheets obviously on day 1 , 3,7, while the $\mathrm{CM}(\mathrm{M} 1)$ inhibited the viability of sheets as compared to control group on

day $3,7{ }^{*} p<0.05,{ }^{* *} p<0.01$ or $\left.* * * p<0.001\right)$. To further explore the influence of condition media on the behavior of cell sheets, researchers changed the media of CM(M0) and $\mathrm{CM}(\mathrm{M} 1)$ groups into $\mathrm{CM}(\mathrm{M} 2)$ media on day 3 then further cultured until day 7, which defined as $\mathrm{CM}(\mathrm{M} 0-\mathrm{M} 2)$ and $\mathrm{CM}(\mathrm{M} 1-\mathrm{M} 2)$ groups. Figures $3 \mathrm{~A}$ and $3 \mathrm{~B}$ show that the viability of both BMSC sheets and pre-vascularized cell sheets in $\mathrm{CM}(\mathrm{M} 0-\mathrm{M} 2)$ or $\mathrm{CM}(\mathrm{M} 1$ M2) was higher than those sheets that were continuously cultured for seven days in CM(M0) or $\mathrm{CM}(\mathrm{M} 1)(* p<0.05, p<0.01$ or $* * * p<0.001)$.

The general view of the BMSC sheet is shown in Figure 3C. Hematoxylin \& eosin (HE) staining images in Figure 3D reveals that the BMSC sheet consisted of five to eight layers 
of cells. Cells resided within their secreted ECM, which provided a physical support for cells and protected both cells and cell-extracellular matrix interactions.

HE staining was performed to observe the morphologies, continuity, and thickness of prevascularized cell sheets cultured in different CMs at the period of 7 days. The images of lateral sections in Figure 4 show that pre-vascularized cell sheets cultured in normal culture medium and $\mathrm{CM}(\mathrm{M} 0)$ had better continuity and more layer of cells than those incubated in $\mathrm{CM}(\mathrm{M} 1)$ and $\mathrm{CM}(\mathrm{M} 2)$. Thickness of pre-vascularized cell sheets was quantified by using Image J (Fig. 3E). Results showed that cell sheets became thicker as time passed and the cell sheets generated in $\mathrm{CM}(\mathrm{M} 1)$ were the thinnest of all groups $(* * p<0.01)$.

\section{Effect of CMs on the network formation of pre-vascularized cell sheets}

The immunofluorescent staining of endothelial marker CD31 was used to observe the process of migration and early network formation on the pre-vascularized cell sheets. As shown in Figure 5A, the HUVECs were orderly arranged and aligned cell-to-cell as vessellike network structures after $24 \mathrm{~h}$ culturing in all 4 groups. There was no significant difference between control and CM groups on first day, but network structures were more distinct in $\mathrm{CM}(\mathrm{M} 1)$ and $\mathrm{CM}(\mathrm{M} 2)$ groups on day 3. As time passed, more intense network branches were observed in control, $\mathrm{CM}(\mathrm{M} 0)$, and $\mathrm{CM}(\mathrm{M} 1)$ groups. Compact CD31 expressions were detected in $\mathrm{CM}(\mathrm{M} 2)$ group after 7 days. In the enlarged images of $\mathrm{CM}(\mathrm{M} 1)$ and CM(M2) on day 7 (Fig. 5 B3, B4), the morphology of HUVEC in CM(M1) was found to be thinner than that in $\mathrm{CM}(\mathrm{M} 2)$. Images of $\mathrm{CM}(\mathrm{M} 0-\mathrm{M} 2)$ and $\mathrm{CM}(\mathrm{M} 1-\mathrm{M} 2)$ groups indicate the effects of dynamic signaling from macrophages secreted for 
angiogenesis, the network structures were enhanced (Fig. 5 B1, B2).

This study further analyzed the concentration of cytokines related to angiogenesis in culture media of HUVECs, BMSC sheets, and pre-vascularized sheets. Figure 6 shows the TNF- $\alpha$, VEGF, PDGF-BB protein levels in the supernatant fluids. Inflammatory cytokines that prime endothelial cells for sprouting, like TNF- $\alpha$, were secreted by M1[21]. The results showed that the content of TNF- $\alpha$ was highest in CM(M1) of the HUVECs group (Fig. 6 $\mathrm{A} 1)$. In the BMSC sheets group, the content of TNF- $\alpha$ in each $\mathrm{CM}$ was comparably higher than that of the normal medium and reduced both in $\mathrm{CM}(\mathrm{M} 1)$ and $\mathrm{CM}(\mathrm{M} 2)$ on day 7(Fig. 6 A2). In the pre-vascularized sheets group, the level of TNF- $\alpha$ in $\mathrm{CM}(\mathrm{M} 1)$ was higher than that of other media (Fig. 6 A3). When the media were switched on day three from $\mathrm{CM}(\mathrm{M} 1)$ to $\mathrm{CM}(\mathrm{M} 2)$, the TNF- $\alpha$ concentration was lower than that in CM1 for seven days $(p<0.05)$. These data indirectly implied that $\mathrm{CM}(\mathrm{M} 1)$ contented the highest level of TNF$\alpha$, while $\mathrm{CM}(\mathrm{M} 0)$ had the lowest level of $\mathrm{TNF}-\alpha$, and the conditioned medium from M2 macrophages may depress the TNF- $\alpha$ in the microenvironment of cell sheet.

Since endothelial cells can produce VEGF, the content of cytokines was relatively high in the experimental groups of HUVECs, and the $\mathrm{CM}(\mathrm{M} 0)$ group was found to have the highest level of VEGF during the seven days (Fig. 6 B1). In the BMSC sheets group, the concentration of VEGF increased gradually over time in all CMs (Fig. 6 B2). As the sources of VEGF were condition media and the HUVECs on cell sheets, the levels of VEGF in each CM condition of pre-vascularized sheets groups were significantly higher than levels of VEGF in BMSC sheets. As shown in Figure 6 B3, the VEGF concentration 
of CM (M0-M2) or CM(M1-M2) was found to be lower than the concentration in $\mathrm{CM}(\mathrm{M} 0)$ or $\mathrm{CM}(\mathrm{M} 1)$ on day 7 . These results suggest that the CMs have little impact of VEGF production of BMSC sheets and the CM(M2) may depress the secretion of VEGF in prevascularized sheets.

Researchers detected the expression of PDGF-BB, which can recruit mesenchymal stem cells and pericytes to stabilize the formation of vasculature[8]. As shown in Figure 6 C13, the levels of PDGF-BB in HUVEC groups increased over time and were hundreds of times higher than those of sheets groups. In the cell sheets groups and pre-vascularized sheets groups, the variation of PDGF-BB content between normal medium and CMs was not obvious. The results imply that the CMs has little influence of the secretion of PDGF$\mathrm{BB}$ in pre-vascularized sheets.

\section{Discussion}

In this study, we explored the effect of CMs derived from different macrophages on the viability and arrangement of HUVECs on BMSC sheets. The human THP-1 cells are typically single and round cells in suspension. After exposure to PMA, THP-1 cells become adherence cells and are differentiated into a macrophage phenotype with distinct morphological changes[19]. It has been widely used to investigate the function and regulation of macrophages in the microenvironment[20,22]. Compared to human primary monocytes like peripheral blood monocytes, the THP-1 cell has a homogeneous genetic background, which minimizes the degree of cell phenotype variability[23]. In addition, the THP-1 cell can be polarized into different subtypes of macrophages by external stimuli, so 
THP-1 cells are widely used in research related to inflammation. Co-cultivation of THP-1 cells with other cell types can lead to a more relevant cell model that more closely mimics the in vivo situation[22, 24].In fact, there is no standardized protocol for using PMA to reliably differentiate THP-1 cells into macrophages. For different experiments, the concentrations of PMA varied from some to several hundred $\mathrm{ng} / \mathrm{ml}$ and differentiation time varied from 24 to $96 \mathrm{~h}$. Based on previous studies, researchers set four concentrations of PMA (5 ng/ml, $50 \mathrm{ng} / \mathrm{ml}, 100 \mathrm{ng} / \mathrm{ml}$, and $200 \mathrm{ng} / \mathrm{ml}$ ) to induce two different concentrations $\left(1.0 \times 10^{5}\right.$ cells $/ \mathrm{ml}$ and $2.5 \times 10^{5}$ cells $\left./ \mathrm{ml}\right)$ of THP- 1 cells into macrophages for 48 or $72 \mathrm{~h}$. According to CCK-8 results and cell densities, $100 \mathrm{ng} / \mathrm{ml}$ of PMA was used to induce 2.5 $\times 10^{5}$ cells $/ \mathrm{ml}$ of THP-1 cells into resting state of macrophages for $48 \mathrm{~h}$, followed by $24 \mathrm{~h}$ of resting. The same stimuli were further used to stimulate the M0 phenotype to activate pro-inflammatory macrophages (LPS plus IFN- $\gamma$ ) and alternatively activated antiinflammatory (IL-4) macrophages with similar concentration used for ex vivo analysis[24]. Specific markers of polarization were evaluated by RT-PCR. Resulting data showed that the M1 markers were upregulated in macrophages stimulated with LPS and INF- $\gamma$, and M2a markers were found to be highly expressed in both resting macrophage and those polarized with IL-4. This may be related to the high concentration of PMA that was used in this study. Previous research indicated that THP-1 may be useful for M1 polarization rather than M2 polarization[25]. Furthermore, the morphology of macrophages was observed using an inverted phase contrast microscope. The M1 phenotype showed a spindle-like shape, while the M2 had a pancake-like or elongated shape. In fact, due to 
different cell lines and culture systems, it is hard to draw a conclusion regarding the macrophage phenotype and morphology[16].

During tissue development and regeneration, macrophages play important roles for vascularization and healing[5]. They can promote angiogenesis by secreting proangiogenic cytokines and behave as support cells for nascent vasculature[26]. However, little attention has been paid to the effects of the microenvironment on endothelial cell viability and alignment on pre-vascularized biomaterials. This study also found that the alignments of HUVECs in condition media appeared to be slender. While HUVECs in normal EBM were found to have a cobblestone-like morphology. The CCK-8 assay implied that the condition media suppressed the viability of HUVECs, but cells were more active in $\mathrm{CM}(\mathrm{M} 1)$ than in $\mathrm{CM}(\mathrm{M} 2)$. At the same time, the Elisa analysis found the level of VEGF in $\mathrm{CM}(\mathrm{M} 1)$ was higher than that in $\mathrm{CM}(\mathrm{M} 2)$. These results may suggest that macrophages may guide the arrangement of endothelial cell through the paracrine pathway[26].

In our previous studies, researchers successfully fabricated a cell sheet using BMSCs as the cell source to explore the network formation of HUVECs on the cell sheet $[13,14]$. Our study further investigated the effects of macrophage secretions on the viability of BMSC sheets and pre-vascularized cell sheets. Results found that $\mathrm{CM}(\mathrm{M} 0)$ and $\mathrm{CM}(\mathrm{M} 2)$ improved the viabilities of BMSC sheets and pre-vascularized sheets, while $\mathrm{CM}(\mathrm{M} 1)$ had the opposite effects. He et al. in 2018 reported M1-polarized macrophages exhibited greater capacity to promote proliferation of BMSCs when compared with other M2-polarized 
macrophages and the control group[16]. However, the subjects in the two studies are different. He et al. focused on the single mesenchymal stem cell, this current research is focused on the BMSC sheets. Both studies found that the cell sheets cultured in a microenvironment with pro-inflammatory macrophages had fewer layers of cells when compared with those generated in other media. This may be because the extracellular matrix formation was depressed in $\mathrm{CM}(\mathrm{M} 1)$. Previous study have demonstrated that macrophages played a fundamental role both in vessel development and in vascularization of tissue-engineered grafts[27]. Dohle et al. added THP-1-derived macrophages into the co-culture system, including endothelial cells and primary osteoblasts and results found a higher number of micro-vessel-like structures formed by endothelial cells[28]. This change correlated with a significantly higher concentration of the pro-angiogenic VEGF and different proinflammatory cytokines, such as IL-6, IL-8, and TNF $\alpha[28]$. In this study, researchers further explored the effects of CMs on the arrangement of HUVECs in the BMSC sheets and the levels of cytokines related to angiogenesis in different CM. According to the results of immunofluorescence staining with the endothelial marker CD31 (red), HUVECs can form a micro-vessel-like network structure on the BMSC sheets, indicating that the cell membrane of BMSCs provided a good environment for the migration and aggregation of HUVECs. In turn, the state of the cell membrane, like varying thickness and textures, affect the network structure formation. The variation in these signals may also be due to the cytokines present in the micro-environment.

In order to further clarify the effect of CMs on the vascular network formation of pre- 
vascularized sheets, ELISA assays were used to detect any changes in the content of cytokines related to angiogenesis in the culture supernatants. Other studies have identified multiple factors that promote the initial and mature stages of angiogenesis. TNF- $\alpha$ is used as a soluble cytokine for macrophage-mediated angiogenesis[29]. VEGF is an effective mitogen of endothelial cells and can induce their germination and proliferation in order to form new immature vascular buds[30]. PDGF-BB promotes the maturation of new blood vessels by activating and recruiting pericytes, stabilizing them, and preventing their regression[31]. The study found that in the culture system of pre-vascularized cell sheets, TNF- $\alpha$ was mainly derived from CMs. The micro vessel-network structures in each group of CMs were denser than those of the control group as observed on day seven. The concentrations of TNF- $\alpha$ in CMs also increased significantly by day seven. According to the resulting data, the VEGF was mainly derived from HUVECs in pre-vascularized sheets. Furthermore, in $\mathrm{CM}(\mathrm{M} 1-\mathrm{M} 2)$ group that condition media were switched after three days from $\mathrm{CM}(\mathrm{M} 1)$ to the $\mathrm{CM}(\mathrm{M} 2)$, the micro-vessel network constructions were more significant as compared to those in media conditioned by a single macrophage phenotype for seven days. This may be due to the fact that the CM(M1-M2) group experienced condition with high levels of TNF- $\alpha$ and VEGF in the first three days, while in the next 4 days, the environment changed into high level of PDGF-BB but low TNF- $\alpha$ and VEGF concentrations. In addition, the sequential release of VEGF and PDGF-BB in scaffold materials can promote angiogenesis in vivo[32]. This indicates that the sequential activation of M1 and M2 macrophages that secrete these two factors will have a significant 
effect.

\section{Conclusion}

In conclusion, researchers systematically detected the effects of condition media from different macrophage phenotypes on the viability and arrangement of HUVECs on Matrigel and BMSC sheets. Data from this study indicate that the fates of pre-vascularized sheets regulated by macrophage secreted factors in the microenvironment and sequentially released cytokines could promote neovascularization and angiogenesis. This supports the idea that the immune system should be considered when designing and using the tissue engineering constructs. However, the differences in condition media may not fully reflect the dynamic situation of the immune system. Further research should be done in order to characterize the direct crosstalk of macrophages and cell sheets by co-culture or Transwell chamber. In addition, since blood vessels need to withstand various pressures and stresses, this research mainly focuses on the relationships between inflammatory cytokines and prevascularized cell sheets. The vessel-like structures formed in this research still require further study before they are used in clinics. 


\section{Figures}

A
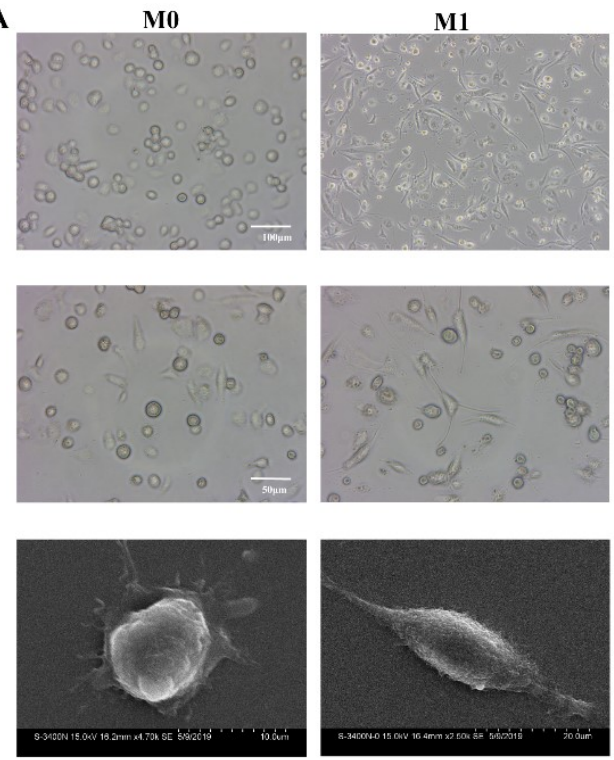

B Table1 PCR primers used in this study.

\begin{tabular}{lll}
\hline Gene & Forward primer & Reward primer \\
\hline GAPDH & ATGGCCTTCCGTGTCCCTACT & TAGCCCACCATGCCCTTGA \\
CD14 & ACGCCAGAACCTTGTGAGC & GCATGGATCTCCACCTCTACTG \\
VEGF-a & ACGGTCCCTCTTGGAATTGG & CTAATCTTCCGGGCTCGGTG \\
PDGF-BB & AGCTGAAAGGGTGGCAACT & CACAGTGAAGTGCTGGCAAC \\
TNF- $\alpha$ & CCACCTCCAGGGACAGGATA & CCTTAAACAGGTTCCCACGC \\
IL-1b & TTCCTTTGGACGGATGGACG & GCGCGCAAAGTATCTCTATCT \\
CCR7 & AGGAAGGCCTAAGGTCCACT & AACACGCAGGACAGGTACAG \\
CD206 & ACTGCGATGCCACAAGTGAA & CCTCGTTACTGTCGCAGGT \\
\hline
\end{tabular}
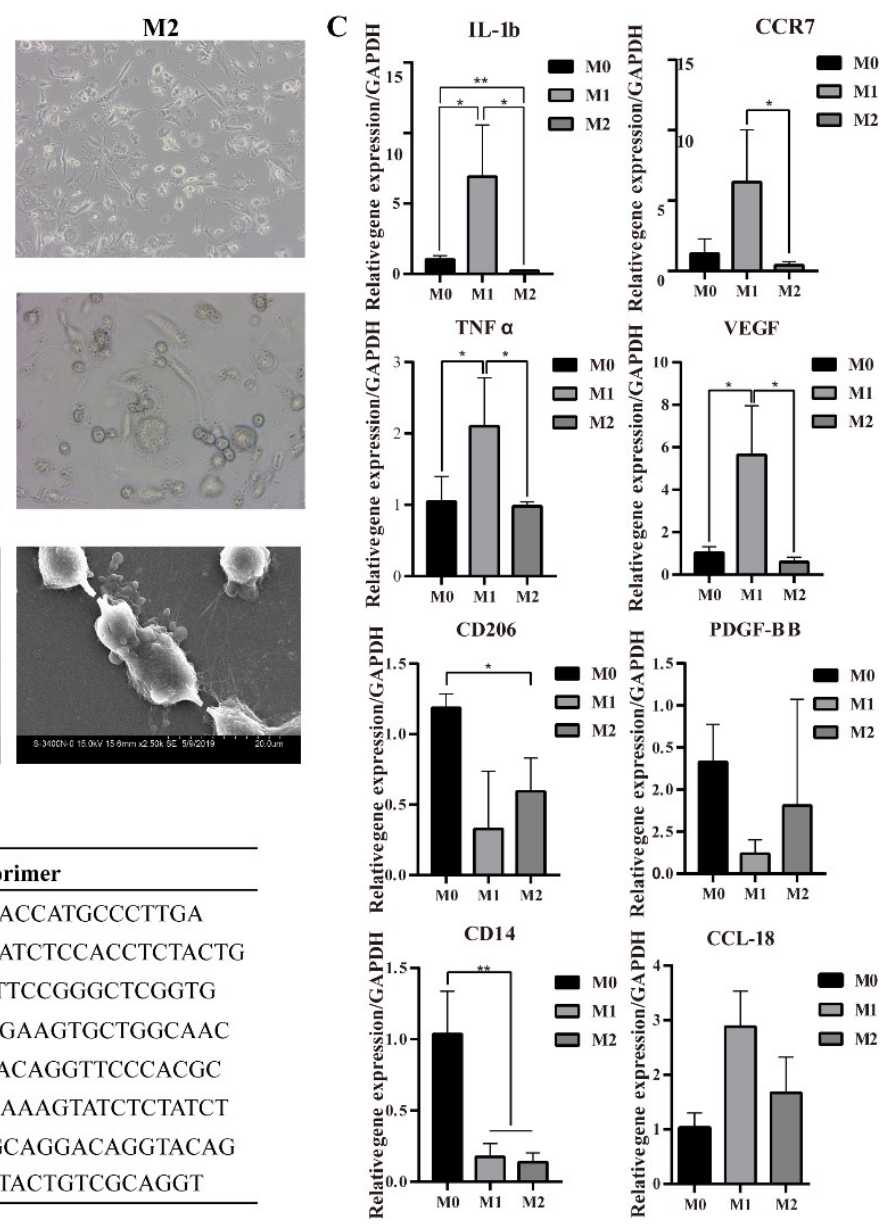

Fig. 1 Differentiation of THP-1 monocytes into macrophages (M0) by culturing in PMA for $48 \mathrm{~h}$ and resting for $24 \mathrm{~h}$ without stimuli. This was followed by polarizing with LPS/IFN- $\gamma$ and IL-4 to get the pro-inflammatory and anti-inflammatory macrophages. A: The morphological appearance of M0, M1, and M2 under the inverted microscope (10X, scale bar $=100 \mu \mathrm{m} ; 20 \mathrm{X}$, scale bar $=50 \mu \mathrm{m})$ and scanning electron microscope. $\mathrm{B}$ : The table of PCR primers used in this study for the identify of M0, M1, M2. C: Gene expression of different markers of M0, M1, and M2 by RT-PCR (CD14 was used as M0 marker; CCR7, 
$T N F-\alpha$, and $V E G F, I L-1 b$ were used as M1 polarized markers; $C D 206$ and $P D G F-B B$, CCL-18 were used as M2 polarized makers; normalized to GAPDH). Data are shown as mean \pm SEM. ${ }^{*} p<0.05,{ }^{* *} p<0.01$.

A
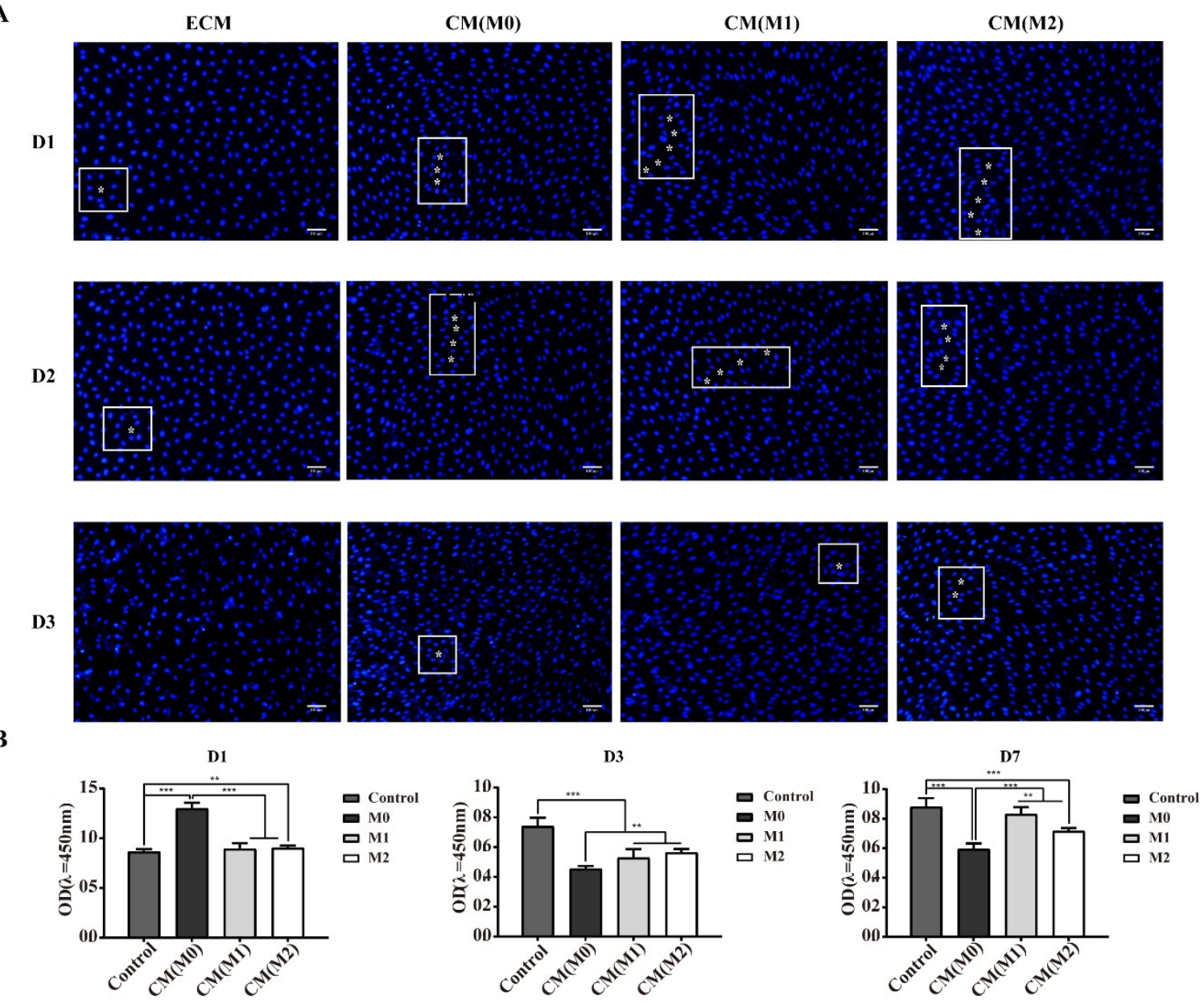

Fig. 2 The effects of the condition media of polarized macrophages on the arrangement and viability of HUVECs, and the normal complete media was used as a control. A: After culturing for 1, 3, and 7 days, arrangement of the nuclei of HUVECs is shown by DAPI staining (Blue fluorescence, scale bar $=100 \mu \mathrm{m}$ ). B: The viability of HUVECs on Matrigel in different condition media evaluated by CCK-8 assays on 1, 3, 7 days. Data are shown as 
mean \pm SEM. ${ }^{* *} p<0.01, * * * p<0.001$

A
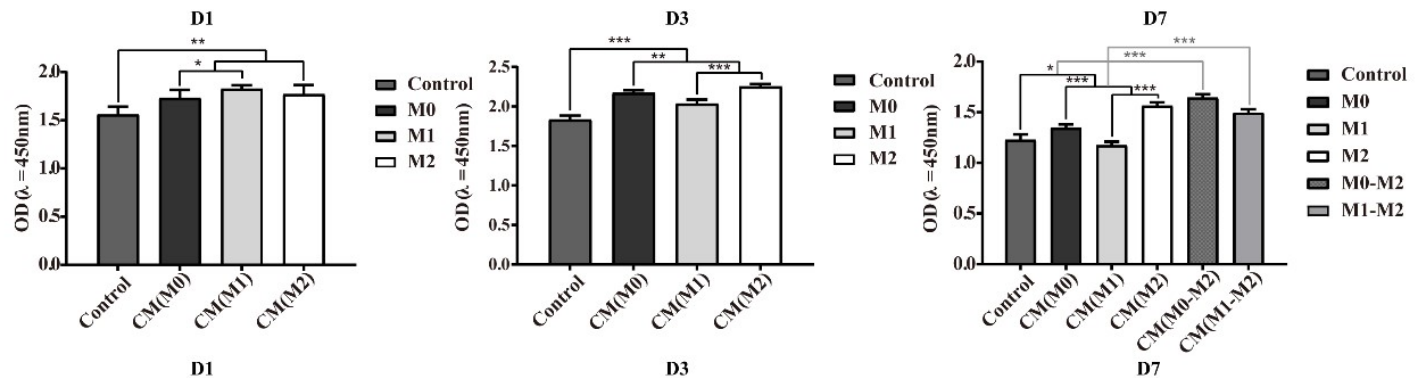

B

D1

D3
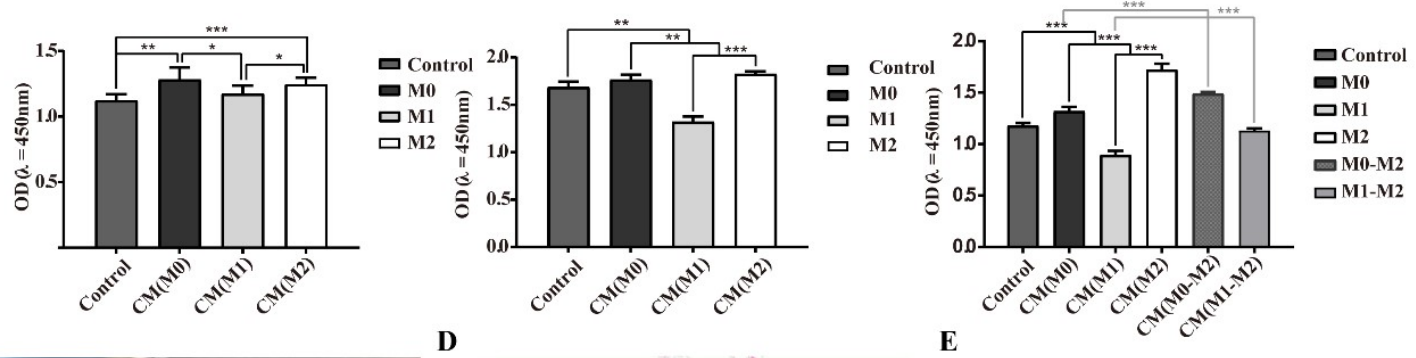

C

D
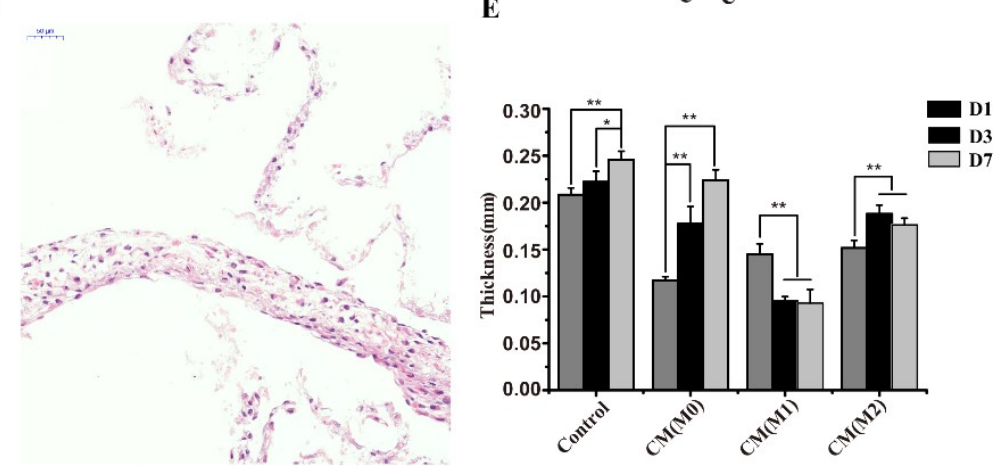

Fig. 3 The effects of the condition media of polarized macrophages on the thickness and viability of BMSC sheets and pre-vascularized cell sheets. A: The viability of BMSC sheets in different condition media evaluated by CCK-8 assays on 1, 3, 7 days. Normal complete media was used as a control. B: The viability of pre-vascularized cell sheets was evaluated by CCK-8 assays in different condition media and the normal complete media on 1,3, and 7 days. C: General view of BMSC sheet. D: The morphology of BMSC sheet observed by HE staining following 2 weeks of cell sheet induction (scale bar= $50 \mu \mathrm{m}$ ). E: The prevascularized cell sheets were cultured in condition media generated by different macrophage phenotypes on 1, 3, and 7 days and quantification of pre-vascularized cell 
sheets thickness using image J. Data are shown as mean \pm SEM. ${ }^{*} p<0.05,{ }^{* *} p<0.01$, $* * * p<0.001$.

D1

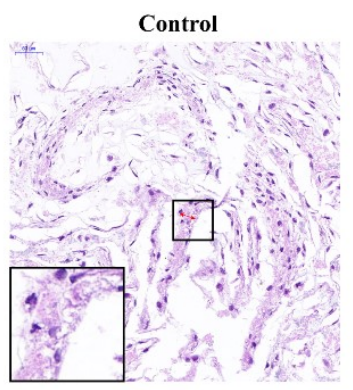

D3

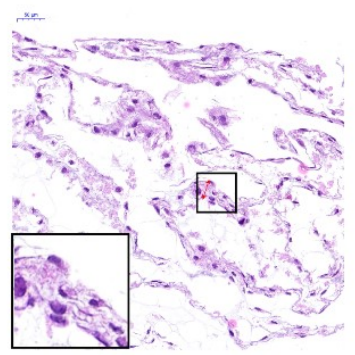

D7

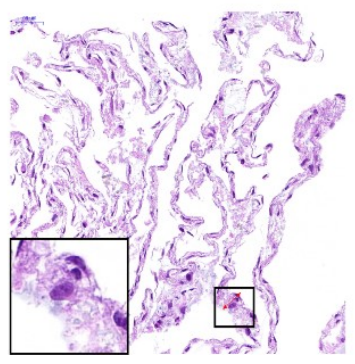

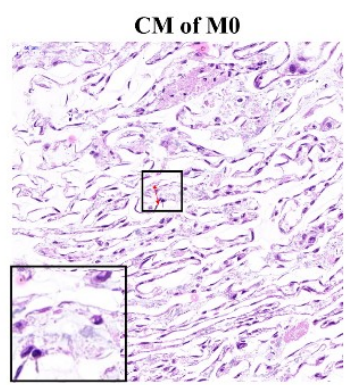
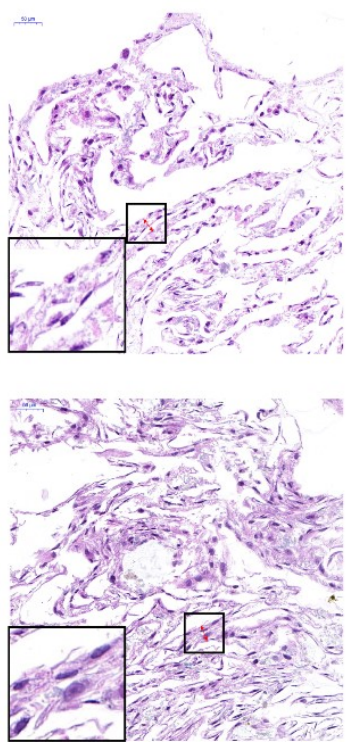
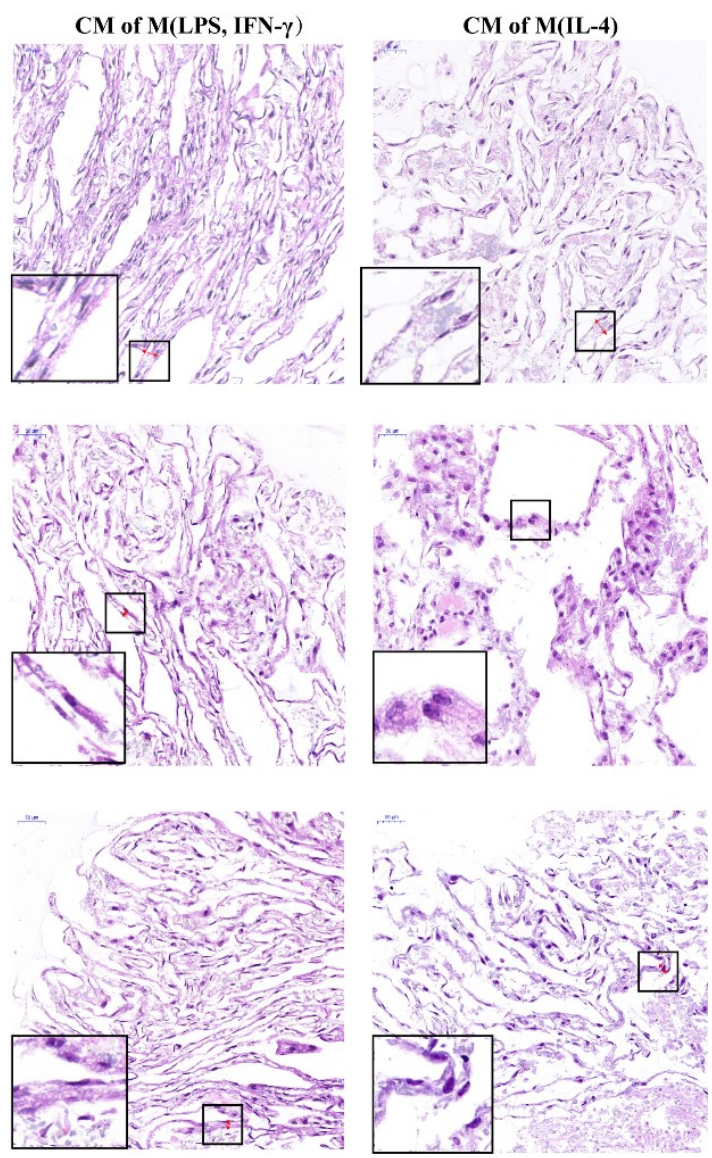

Fig. 4 The morphologies of pre-vascularized cell sheets cultured in different condition media were observed by HE staining on 1, 3, and 7 days, and the normal culture medium was used as a control (scale bar $=50 \mathrm{v} \mu \mathrm{m})$. 


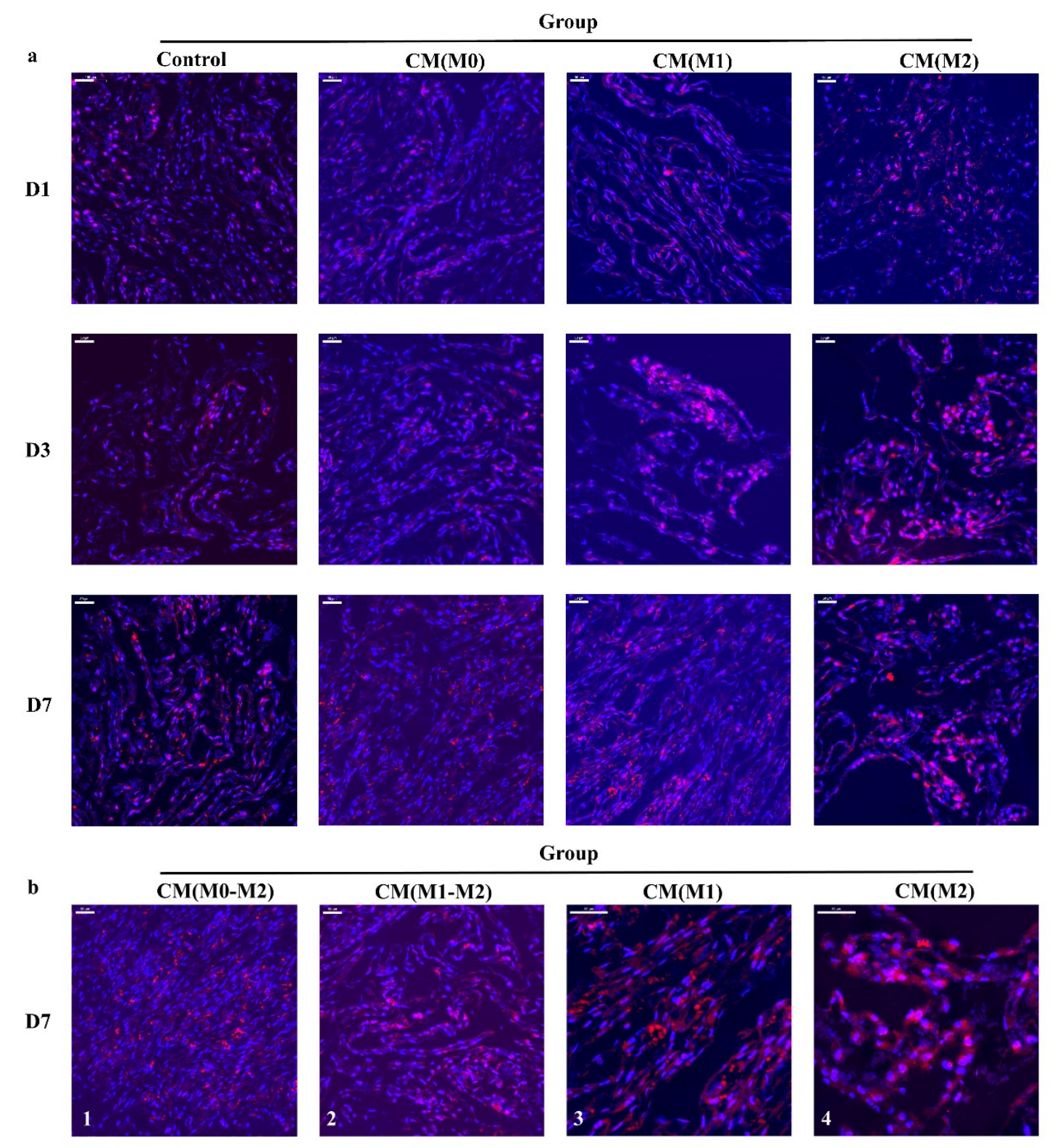

Fig. 5 Immunofluorescent staining for the HUVECs maker CD31 on pre-vascularized cell sheets. A: The process of the vascular-like network formation on cell sheets in different macrophages condition media on 1,3 , and 7 days (scale bar $=50 \mu \mathrm{m}$ ). B: After culturing for 7 days, the alignment of HUVECs on cell sheets was indicated by the red fluorescent of CD31 (B1-2, scale bar= $50 \mu \mathrm{m} ; \mathrm{B} 3-4$, scale bar $=100 \mu \mathrm{m})$. 

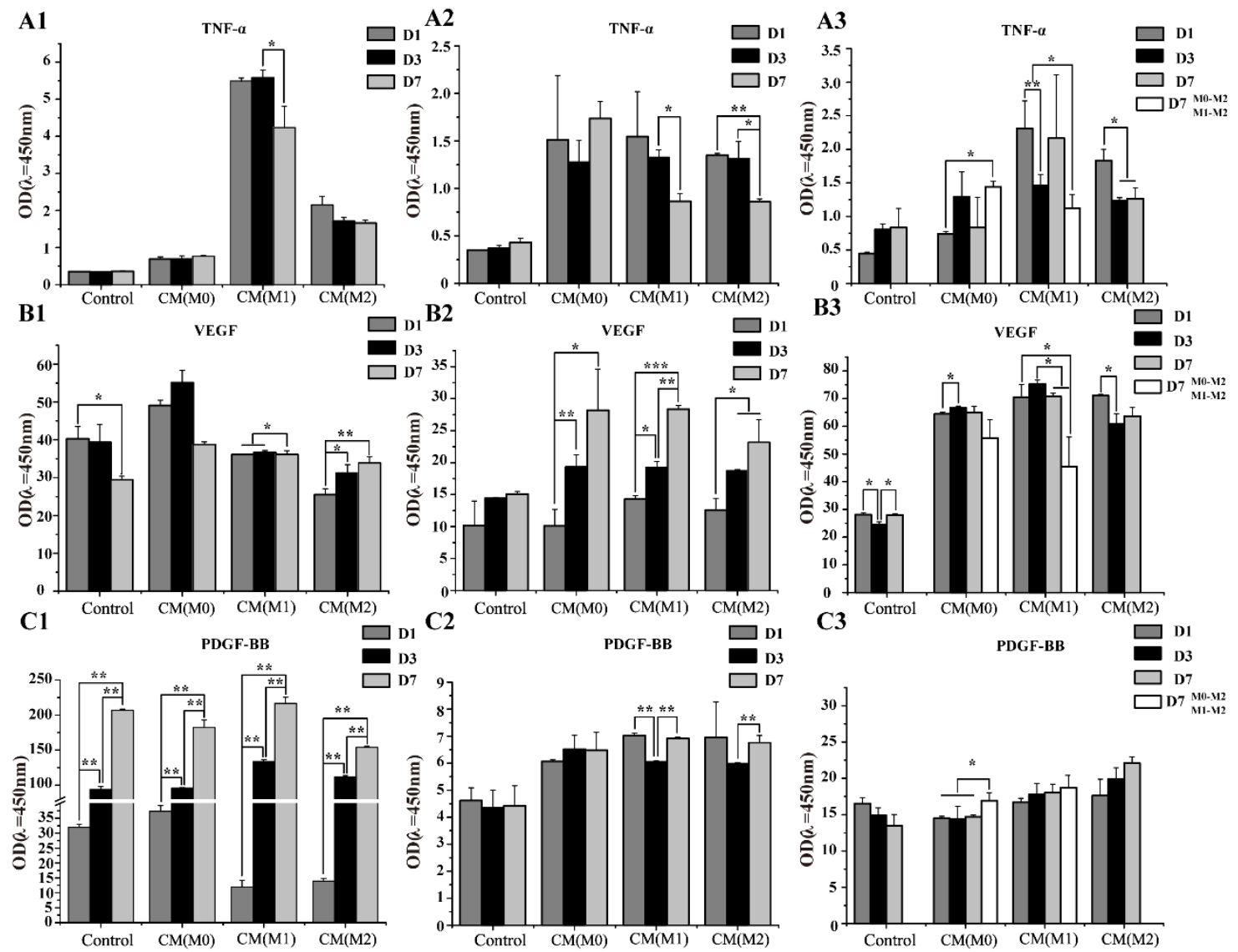

Fig. 6 ELISA of different macrophages condition media for levels of vascular formation related protein secretion. The histograms of A1, B1, and C1 present for HUVEC culture systems; the histograms of $\mathrm{A} 2, \mathrm{~B} 2$, and $\mathrm{C} 2$ present for BMSC sheet culture systems; the histograms of A3, B3, and C3 presents for pre-vascularized sheets. Data are shown as mean \pm SEM. $* p<0.05, * * p<0.01, * * * p<0.001$. 


\section{Abbreviations}

HUVECs: Human umbilical vein endothelial cells

BMSC: Bone marrow mesenchymal stem cell

THP-1: Human monocytic leukemia cell line

M0: The resting state of macrophage

M1: Pro- inflammatory macrophage

M2: Anti-inflammatory macrophage

PMA: Phorbol 12-myristate 13-acetate

TNF- $\alpha$ : Tumor necrosis factor alpha

VEGF: Vascular endothelial growth factor

PDGF-BB: Platelet-derived growth factor

CM: Conditioned media

ATCC: American Type Culture Collection

DEPC: Diethyl pyrocarbonate

RT-PCR: Quantitative real-time polymerase chain reaction

MMP: Matrix metalloprotinase

IL: Interleukin

GAPDH: Glyceraldehyde-3-phosphate dehydrogenase

MSCM: mesenchymal stem cell medium

EBM: Endothelial Basal Medium

FBS: Fetal bovine serum 
DPBS: Dulbecco's phosphate buffered saline

OD: Optical density

CD31: Platelet-endothelial cell adhesion molecule

HE: Hematoxylin \& eosin

\section{References}

1. Vishwakarma A, Bhise NS, Evangelista MB, Rouwkema J, Dokmeci MR, Ghaemmaghami AM, et al. Engineering Immunomodulatory Biomaterials To Tune the Inflammatory Response. Trends in biotechnology. 2016;34(6):470-82.

2. Mariani E, Lisignoli G, Borzi RM, Pulsatelli L. Biomaterials: Foreign Bodies or Tuners for the Immune Response? Int J Mol Sci. 2019;20(3).

3. Graney PL, Ben-Shaul S, Landau S, Bajpai A, Singh B, Eager J, et al. Macrophages of diverse phenotypes drive vascularization of engineered tissues. Sci Adv. 2020;6(18).

4. Mohammadi R, Cao R, Rodriguez S, Tolsma D, Lakey J. Immunomodulatory Stem Cells to Alleviate the Foreign Body Response Against Implanted Biomaterials. Transplantation. 2019;103(9):S9 -S10.

5. Julier Z, Park AJ, Briquez PS, Martino MM. Promoting tissue regeneration by modulating the immune system. Acta biomaterialia. 2017;53:13-28.

6. Morris AH, Stamer DK, Kyriakides TR. The host response to naturally-derived extracellular matrix biomaterials. Seminars in immunology. 2017;29:72-91.

7. Minutti CM, Knipper JA, Allen JE, Zaiss DM. Tissue-specific contribution of macrophages to wound healing. Seminars in cell \& developmental biology. 2017;61:3-11.

8. Spiller KL, Anfang RR, Spiller KJ, Ng J, Nakazawa KR, Daulton JW, et al. The role of macrophage phenotype in vascularization of tissue engineering scaffolds. Biomaterials. 2014;35(15):4477-88.

9. Huang SQ, Yue Y, Peng KN, Huang XL, Li HY, Hou J, et al. Conditioned medium from M2b macrophages modulates the proliferation, migration, and apoptosis of pulmonary artery smooth muscle cells by deregulating the PI3K/Akt/FoxO3a pathway. Peerj. 2020;8.

10. Martin KE, Garcia AJ. Macrophage phenotypes in tissue repair and the foreign body response: Implications for biomaterial-based regenerative medicine strategies. Acta biomaterialia. 2021;133:416.

11. Sakaguchi K, Shimizu T, Horaguchi S, Sekine H, Yamato M, Umezu M, et al. In vitro engineering of vascularized tissue surrogates. Scientific reports. 2013;3:1316.

12. Bou-Ghannam S, Kim K, Grainger DW, Okano T. 3D cell sheet structure augments mesenchymal stem cell cytokine production. Scientific reports. 2021;11(1):8170.

13. Zhang D, Gao P, Li Q, Li J, Li X, Liu X, et al. Engineering biomimetic periosteum with $\beta-\mathrm{TCP}$ scaffolds to promote bone formation in calvarial defects of rats. Stem cell research \& therapy. 2017;8(1):134.

14. Chen J, Zhang D, Li Q, Yang D, Fan Z, Ma D, et al. Effect of different cell sheet ECM 
microenvironment on the formation of vascular network. Tissue \& cell. 2016;48(5):442 -51.

15. Sukho P, Hesselink JW, Kops N, Kirpensteijn J, Verseijden F, Bastiaansen-Jenniskens YM. Human Mesenchymal Stromal Cell Sheets Induce Macrophages Predominantly to an Anti-Inflammatory Phenotype. Stem cells and development. 2018;27(13):922-34.

16. He XT, Li X, Yin Y, Wu RX, Xu XY, Chen FM. The effects of conditioned media generated by polarized macrophages on the cellular behaviours of bone marrow mesenchymal stem cells. Journal of cellular and molecular medicine. 2018;22(2):1302-15.

17. Lund ME, To J, O'Brien BA, Donnelly S. The choice of phorbol 12-myristate 13-acetate differentiation protocol influences the response of THP-1 macrophages to a pro-inflammatory stimulus. Journal of immunological methods. 2016;430:64-70.

18. Maess MB, Wittig B, Cignarella A, Lorkowski S. Reduced PMA enhances the responsiveness of transfected THP-1 macrophages to polarizing stimuli. Journal of immunological methods. 2014;402(12):76-81.

19. Tsuchiya S, Kobayashi Y, Goto Y, Okumura H, Nakae S, Konno T, et al. Induction of maturation in cultured human monocytic leukemia cells by a phorbol diester. Cancer research. 1982;42(4):1530-6.

20. Freytes DO, Kang JW, Marcos-Campos I, Vunjak-Novakovic G. Macrophages modulate the viability and growth of human mesenchymal stem cells. Journal of cellular biochemistry. 2013;114(1):220-9.

21. Sainson RC, Johnston DA, Chu HC, Holderfield MT, Nakatsu MN, Crampton SP, et al. TNF primes endothelial cells for angiogenic sprouting by inducing a tip cell phenotype. Blood. 2008;111(10):4997 5007.

22. Qin Z. The use of THP-1 cells as a model for mimicking the function and regulation of monocytes and macrophages in the vasculature. Atherosclerosis. 2012;221(1):2-11.

23. Rogers PD, Thornton J, Barker KS, McDaniel DO, Sacks GS, Swiatlo E, et al. Pneumolysindependent and -independent gene expression identified by cDNA microarray analysis of THP-1 human mononuclear cells stimulated by Streptococcus pneumoniae. Infection and immunity. 2003;71(4):2087-94.

24. Chanput W, Mes JJ, Wichers HJ. THP-1 cell line: an in vitro cell model for immune modulation approach. International immunopharmacology. 2014;23(1):37-45.

25. Shiratori H, Feinweber C, Luckhardt S, Linke B, Resch E, Geisslinger G, et al. THP-1 and human peripheral blood mononuclear cell-derived macrophages differ in their capacity to polarize in vitro. Molecular immunology. 2017;88:58-68.

26. Ng WL, Wang S, Yeong WY, Naing MW. Skin Bioprinting: Impending Reality or Fantasy? Trends in biotechnology. 2016;34(9):689-99.

27. Spiller KL, Freytes DO, Vunjak-Novakovic G. Macrophages modulate engineered human tissues for enhanced vascularization and healing. Annals of biomedical engineering. 2015;43(3):616-27.

28. Dohle E, Bischoff I, Böse T, Marsano A, Banfi A, Unger RE, et al. Macrophage-mediated angiogenic activation of outgrowth endothelial cells in co-culture with primary osteoblasts. European cells \& materials. 2014;27:149-64; discussion 64-5.

29. Leibovich SJ, Polverini PJ, Shepard HM, Wiseman DM, Shively V, Nuseir N. Macrophage-induced angiogenesis is mediated by tumour necrosis factor-alpha. Nature. 1987;329(6140):630-2. 
30. Ferrara N. VEGF: an update on biological and therapeutic aspects. Current opinion in biotechnology. 2000;11(6):617-24.

31. Song N, Huang Y, Shi H, Yuan S, Ding Y, Song X, et al. Overexpression of platelet-derived growth factor-BB increases tumor pericyte content via stromal-derived factor-1alpha/CXCR4 axis. Cancer research. 2009;69(15):6057-64.

32. Spiller KL, Vunjak-Novakovic G. Clinical translation of controlled protein delivery systems for tissue engineering. Drug delivery and translational research. 2015;5(2):101-15.

\section{Declarations}

Ethics approval and consent to participate

Not applicable

Consent for publication

Not applicable

Availability of data and materials

Not applicable

Competing interests

The authors declare that they have no competing interests.

Funding

This work was supported by grants National Natural Science Foundation of China (Nos. 81670969).

Authors' contributions

LR, PZ and SL designed the experiments. SL, JS, LR and XL conducted the experiments. PZ supervised the research. SL, JS, LR, JL contributed to data analysis and figure preparation, SL and LR wrote the manuscript. PZ, DM and LR revised the manuscript and provided the funding. All authors read and approved the final manuscript.

Acknowledgements

Not applicable

Authors' information

West China School of Stomatology, Sichuan University, No.14, Section 3, Renmin South Road, Wuhou District, Chengdu,610065, PR China

Siqi Long

School and Hospital of Stomatology, Lanzhou University, No.222 Tianshui South Road, Chengguan District, Lanzhou 730000, Gansu Province, PR China.

Liling Ren, Pin Zhou, Lina Ren, Jiaxin Liu 
Department of Laboratory Animal Centre, Changzhi Medical College, No.161 Liberation East Street, Luzhou District, Changzhi 046000, Shanxi Province, PR China

Jiamin Shi

Hangzhou Zhongyi Dental Clinic, No.170, Zhonghe Middle Road, Shangcheng Distict, Hangzhou, Zhejiang, 310000, PR China

Xiaoning Liu

Department of Oral and Maxillofacial Surgery, the 940th Hospital of Joint Logistics Support Force of PLA, Lanzhou, Gansu, 730052, PR China

Dongyang Ma

Corresponding authors

Correspondence to Pin Zhou or Liling Ren 


\section{Supplementary Files}

This is a list of supplementary files associated with this preprint. Click to download.

- Surpportinginformation.docx 